\title{
Convergence Analysis for Mixed Finite Element Method of Positive Semi-definite Problems
}

\author{
Yirang Yuan ${ }^{1}$, Changfeng $\mathrm{Li}^{1,2}$ \\ ${ }^{1}$ Institute of Mathematics, Shandong University, Jinan, China \\ ${ }^{2}$ School of Economics, Shandong University, Jinan, China
}

Correspondence: Yirang Yuan, Institute of Mathematics, Shandong University, Jinan, ShandaNanlu 27, China. Tel: 86531-8836-4732. E-mail: yryuan@sdu.edu.cn

$\begin{aligned} & \text { Received: January 20, } 2017 \quad \text { Accepted: March 10, } 2017 \quad \text { Online Published: May 9, } 2017 \\ & \text { doi:10.5539/jmr.v9n3p14 }\end{aligned}$ URL: https://doi.org/10.5539/jmr.v9n3p14

The research is financed by the National Natural Science Foundation of China (Grant No. 11271231), Natural Science Foundation of Shandong Province (Grant No. ZR2016AM08), National Tackling Key Problems Program (Grant Nos. 2011ZX05052, 2011ZX05011-004), NSAF (Grant No. U1430101)

\begin{abstract}
A mixed element-characteristic finite element method is put forward to approximate three-dimensional incompressible miscible positive semi-definite displacement problems in porous media. The mathematical model is formulated by a nonlinear partial differential system. The flow equation is approximated by a mixed element scheme, and the pressure and Darcy velocity are computed at the same time. The concentration equation is treated by the method of characteristic finite element, where the convection term is discretized along the characteristics and the diffusion term is computed by the scheme of finite element. The method of characteristics can confirm strong computation stability at the sharp fronts and avoid numerical dispersion and nonphysical oscillation. Furthermore, a large step is adopted while small time truncation error and high order accuracy are obtained. It is an important feature in numerical simulation of seepage mechanics that the mixed volume element can compute the pressure and Darcy velocity simultaneously and the accuracy of Darcy velocity is improved one order. Using the form of variation, energy method, $L^{2}$ projection and the technique of priori estimates of differential equations, we show convergence analysis for positive semi-definite problems. Then a powerful tool is given to solve international famous problems.
\end{abstract}

Keywords: three-dimensional incompressible miscible displacement, positive semi-definite problem, mixed element with characteristic finite element, error estimate in $L^{2}$-norm

\section{Introduction}

The incompressible miscible positive semi-definite displacement problem in porous media consists of two partial different equations: an elliptic equation for the pressure, a convection-diffusion equation for the concentration, where the concentration equation has strong hyperbolic feature (Douglas, 1983; Dougals, Ewing \& Wheeler, 1983; Ewing, Russell \& Wheeler, 1984; Russell, 1985),

$$
\begin{gathered}
-\nabla \cdot\left(\frac{\kappa(X)}{\mu(X)}(\nabla p-\gamma(c) \nabla d(X))\right) \equiv \nabla \cdot \mathbf{u}=q, X \in \Omega, t \in J=(0, T], \\
\mathbf{u}=-\frac{\kappa(X)}{\mu(X)}(\nabla p-\gamma(c) \nabla d(X)), X \in \Omega, t \in J . \\
\phi \frac{\partial c}{\partial t}+\mathbf{u} \cdot \nabla c-\nabla \cdot(\mathbf{D}(X, \mathbf{u}) \nabla c)=(\tilde{c}-c) \tilde{q}, X \in \Omega, t \in J, \\
\mathbf{u} \cdot v=(\mathbf{D}(X, \mathbf{u}) \nabla c) \cdot v=0, X \in \partial \Omega, t \in J, \\
c(X, 0)=c_{0}(X), X \in \Omega .
\end{gathered}
$$

$\Omega$ denotes a bounded domain of $R^{3}$, and $v$ is the normal outer vector to the boundary surface, denoted by $\partial \Omega$. The pressure, $p(X, t)$, Darcy velocity, $\mathbf{u}=\left(u_{1}, u_{2}, u_{3}\right)^{T}$ and the concentration of water, $c(X, t)$, are objective functions. $q(X, t)$, the quantity, is greater than zero at injection wells and is less than zero at production wells, and $\tilde{q}=\max \{q, 0\} . \phi(X)$ is the porosity of rock, and $\kappa(X)$ is absolute permeability. $\mu(c)$, the viscosity of mixture, depends on $c$. $\tilde{c}$, the concentration of injected fluid, is equal to $c$ at production wells, $\tilde{c}=c . \gamma(c)$ and $d(X)=(0,0, z)^{T}$ denote the gravitational coefficient and 
vertical coordinates, respectively. The diffusion matrix, $\mathbf{D}(X, \mathbf{u})$, is generally defined by (Yuan \& Han, 2008; Yuan, Wang \& Han, 2010),

$$
\mathbf{D}(X, \mathbf{u})=D_{m}(X) \mathbf{I}+\alpha_{l}|\mathbf{u}|^{\beta}\left(\begin{array}{ccc}
\hat{u}_{x}^{2} & \hat{u}_{x} \hat{u}_{y} & \hat{u}_{x} \hat{u}_{z} \\
\hat{u}_{x} \hat{u}_{y} & \hat{u}_{y}^{2} & \hat{u}_{y} \hat{u}_{z} \\
\hat{u}_{x} \hat{u}_{z} & \hat{u}_{y} \hat{u}_{z} & \hat{u}_{z}^{2}
\end{array}\right)+\alpha_{t} \mid \mathbf{u}^{\beta}\left(\begin{array}{ccc}
\hat{u}_{y}^{2}+\hat{u}_{z}^{2} & -\hat{u}_{x} \hat{u}_{y} & -\hat{u}_{x} \hat{u}_{z} \\
-\hat{u}_{x} \hat{u}_{y} & \hat{u}_{x}^{2}+\hat{u}_{z}^{2} & -\hat{u}_{y} \hat{u}_{z} \\
-\hat{u}_{x} \hat{u}_{z} & -\hat{u}_{y} \hat{u}_{z} & \hat{u}_{x}^{2}+\hat{u}_{y}^{2}
\end{array}\right) .
$$

$D_{m}$ is the molecular diffusivity. I denotes a $3 \times 3$ unit matrix. $\alpha_{l}$ and $\alpha_{t}$ are the longitudinal and the transverse dispersivities, respectively. $\hat{u}_{x}, \hat{u}_{y}, \hat{u}_{z}$ denote three direction cosines of $\mathbf{u}$. Generally speaking, the symbol, $\beta \geq 2$, is a positive constant. The mathematical model is usually used to describe numerical simulation of oil reservoir and contaminant-transport problem, and the diffusion matrix is supposed to be positive definite. While in actual numerical simulation applications such as oil-gas resources basin assessment (Yuan \& Han, 2008; Yuan, Wang \& Han, 2010) and numerical computation of enhanced (chemical) oil recovery (Yuan, Cheng, Yang \& Li, 2014,2015), the diffusion matrix is only positive semi-definite (Dawson, Russell \& Wheeler, 1989; Ewing, 1983; Yuan, 2013),

$$
\mathbf{D}(X, \mathbf{u}) \geq 0 .
$$

The present paper mainly considers a positive semi-definite problem, and the discussion gives more theoretical reference in terms of mathematics and mechanics (Ewing, 1983; Yuan, 2013).

Oil-water two phase seepage displacement is a primary topic in numerical simulation of oil reservoir. For two-dimensional positive definite problems, Douglas and Russell presented well-known numerical methods such as characteristic finite difference and characteristic finite element (Russell, 1985; Douglas, 1983). Douglas, Ewing and Wheeler put forward the method of mixed element (Douglas, Ewing \& Wheeler, 1983), and Ewing, Russell, Wheeler discussed the characteristicsmixed element (Ewing, Russell \& Wheeler, 1984). The above arguments were based on the positive definite assumption, but the diffusion matrix was only positive semi-definite in some actual applications (Dawson, Russell \& Wheeler, 1989; Ewing, 1983; Yuan \& Han, 2008; Yuan, Wang \& Han, 2010; Yuan, 2013; Yuan, Cheng, Yang \& Li, 2014,2015). Therefore, the framework of theoretical analysis is not feasible. It is hard and difficult to show convergence analysis of semidefinite problem. The characteristic finite element method was presented by Dawson (Dawson, Russell \& Wheeler, 1989). For three-dimensional positive semi-definite problems, Yuan discussed characteristic finite element and characteristic finite difference (Yuan, 1997,1999). Based on the above discussions, we present a method of mixed element-characteristic finite element to simulate three-dimensional incompressible miscible positive semi-definite displacement problem of (1)(4). The flow equation is treated by a conservative mixed element method, and the pressure and Darcy velocity are obtained at the same time. The characteristic finite element is used to solve the concentration equation, where the convection term is discretized along the characteristics and the diffusion term is approximated by the finite element method. The characteristics can confirm strong stability at the fronts and can avoid numerical dispersion. Moreover, it can adopt large spatial step while computational accuracy is not decreased. More important in numerical simulation of seepage mechanics, the pressure and Darcy velocity are obtained simultaneously by using the scheme of mixed element and the computational accuracy of Darcy velocity is developed one order. Using variation form, energy method, $L^{2}$ projection and theoretical framework of priori estimate, we show convergence analysis in $L^{2}$ norm. Then the well-known difficult problem is solved numerically, and a basic theoretical reference is given for actual numerical simulations.

common symbols and notations of Sobolev space are adopted. Suppose that the problem of (1)-(4) is regular,

$$
\left\{\begin{array}{l}
c \in L^{\infty}\left(H^{l+1}\right) \cap H^{1}\left(H^{l+1}\right) \cap L^{\infty}\left(W_{\infty}^{1}\right) \cap H^{2}\left(L^{2}\right) \\
p \in L \infty\left(H^{k+1}\right) \\
\mathbf{u} \in L^{\infty}\left(H^{k+1}(\mathrm{div})\right) \cap L^{\infty}\left(W_{\infty}^{1}\right) \cap W_{\infty}^{1}\left(L^{\infty}\right) \cap H^{2}\left(L^{2}\right)
\end{array}\right.
$$

where $l \geq 3, k \geq 1$.

And suppose that the problem is positive semi-definite

$$
\text { (C) } 0<a_{*} \leq \frac{k(X)}{\mu(c)} \leq a^{*}, 0<\phi_{*} \leq \phi(X) \leq \phi^{*}, \mathbf{D}(X, \mathbf{u}) \geq 0,
$$

where $a_{*}, a^{*}, \phi_{*}$ and $\phi^{*}$ are positive constants.

In this paper, the symbols $K$ and $\varepsilon$ denote a generic positive constant and a generic small positive number, respectively. They have different definitions at different places. 


\section{The Computation Program}

\subsection{The Mixed Element for the Pressure}

The form of variation is discussed. Let $H(\operatorname{div} ; \Omega)$ denote a space consisting of vector functions, $\mathbf{v} \in L^{2}(\Omega)^{3}$, satisfying $\nabla \cdot \mathbf{v} \in L^{2}(\Omega)$, then define

$$
V=H(\operatorname{div} ; \Omega) \bigcap\{\mathbf{v} \cdot v=0 \text { on the boundary } \partial \Omega\} .
$$

The pressure $p(X, t)$ is determined only except an additive constant. For simplicity, consider a factor space

$$
W=L^{2}(\Omega) /\{\varphi \equiv \text { const. on the boundary } \partial \Omega\} .
$$

For $\alpha, \beta \in V, \varphi \in W$ and $\theta \in L^{\infty}(\Omega)$, define the following bilinear function

$$
\begin{aligned}
& \mathcal{A}(\theta, \alpha, \beta)=\left(\frac{\mu(\theta)}{k} \alpha, \beta\right), \\
& \mathcal{B}(\alpha, \varphi)=-(\nabla \cdot \alpha, \varphi) .
\end{aligned}
$$

Then the pressure equation is equivalent to a family of saddle point problems:

$$
\begin{aligned}
& \mathcal{A}(c, \mathbf{u}, \mathbf{v})+\mathcal{B}(\mathbf{v}, p)=(\gamma(c) \nabla d, \mathbf{v}), \forall v \in V, \\
& \mathcal{B}(\mathbf{u}, w)=-(q, w), \forall w \in W .
\end{aligned}
$$

The problem of (11) is considered. Let $h_{p}>0$ be the spatial step for the pressure, and let $J_{h_{p}}$ be a quasi-regular partition of $\Omega$, consisting of tetrahedrons or cubes with the greatest diameter at most $h_{p}$. Let $V_{h} \times W_{h} \subset V \times W$ be a Raviar-Thomas space on the partition (Raviart \& Thomase, 1977; Thomase, 1977), with the index $k$ and the approximation $O\left(h_{p}^{k+1}\right)$, whose approximations satisfy

$$
\begin{aligned}
& \left(A_{p}\right) \quad\left\{\begin{array}{l}
\inf _{\mathbf{v}_{h} \in V_{h}}\left\|\mathbf{v}-\mathbf{v}_{h}\right\|_{L^{2}(\Omega)^{3}} \leq K\|\mathbf{v}\|_{H^{k+1}(\Omega)^{3}} h_{p}^{k+1}, \\
\inf _{\mathbf{v}_{h} \in V_{h}}\left\|\mathbf{v}-\mathbf{v}_{h}\right\|_{V} \leq K\left\{\|\mathbf{v}\|_{H^{k+1}(\Omega)^{3}}+\|\nabla \cdot \mathbf{v}\|_{H^{k+1}(\Omega)}\right\} h_{p}^{k+1}, \\
\inf _{w_{h} \in W_{h}}\left\|w-w_{h}\right\| \leq K\|w\|_{H^{k+1}(\Omega)} h_{p}^{k+1},
\end{array}\right. \\
& \left(I_{p}\right) \quad\|w\|_{L^{\infty}(\Omega)} \leq K h_{p}^{-3 / 2}\|w\|_{L^{2}(\Omega)},\|w\|_{H^{1}(\Omega)} \leq K h_{p}^{-1}\|w\|_{L^{2}(\Omega)} .
\end{aligned}
$$

Introduce elliptic projection of $(\mathbf{u}, p)$ to find $\left(\tilde{\mathbf{u}}_{h}, \tilde{p}_{h}\right):[0, T] \rightarrow V_{h} \times W_{h}$ such that

$$
\begin{aligned}
& \mathcal{A}\left(c, \tilde{\mathbf{u}}_{h}, \mathbf{v}\right)+\mathcal{B}\left(\mathbf{v}, \tilde{p}_{h}\right)=(\gamma(c) \nabla d, \mathbf{v}), \forall \mathbf{v} \in V, \\
& \mathcal{B}\left(\tilde{\mathbf{u}}_{h}, w\right)=-(q, w), \forall w \in W,
\end{aligned}
$$

where $c$ is the exact concentration.

It is seen that in the references (Douglas, Ewing \& Wheeler, 1983; Ewing, Russell \& Wheeler, 1984) the solution $\left(\tilde{\mathbf{u}}_{h}, \tilde{p}_{h}\right)$ exists solely and is estimated as follows

$$
\left\|\tilde{\mathbf{u}}_{h}-\mathbf{u}\right\|_{L^{\infty}(H(\operatorname{div}))}+\left\|\tilde{p}_{h}-p\right\|_{L^{\infty}\left(L^{2}\right)} \leq K h_{p}^{k+1} .
$$

Then it follows from (14) and $\left(I_{p}\right)$, for $k \geq 1$,

$$
\|\tilde{\mathbf{u}}\|_{L^{\infty}\left(L^{\infty}\right)} \leq K .
$$

The mixed element scheme is constructed. When the approximate concentration $c_{h}$ at $t \in J$ is known, then $\left(\mathbf{u}_{h}, p_{h}\right) \in$ $V_{h} \times W_{h}$ is aimed to find

$$
\begin{aligned}
& \mathcal{A}\left(c_{h}, \mathbf{u}_{h}, \mathbf{v}\right)+\mathcal{B}\left(\mathbf{v}, p_{h}\right)=\left(\gamma\left(c_{h}\right) \nabla d, \mathbf{v}\right), \forall \mathbf{v} \in V_{h}, \\
& \mathcal{B}\left(\mathbf{u}_{h}, w\right)=-(q, w), \forall w \in W_{h} .
\end{aligned}
$$

It has been proved that numerical solutions of (16) exist solely (Brezzi, 1974). Using (14) and (15),

$$
\left\|\mathbf{u}_{h}-\tilde{\mathbf{u}}_{h}\right\|_{H(\mathrm{div})}+\left\|p_{h}-\tilde{p}_{h}\right\| \leq K\left(1+\left\|\tilde{\mathbf{u}}_{h}\right\|_{L^{\infty}}\right)\left\|c-c_{h}\right\|_{L^{2}}
$$

The concentration equation (2) is discretized later. 


\subsection{The Finite Element Approximation for the Concentration}

For convenience to interpret the approximation of concentration, we suppose that Darcy velocity $\mathbf{u}=\left(u_{1}, u_{2}, u_{3}\right)^{T}$ is given. The procedures are constructed by combining the discretization of characteristics and the approximation of finite element.

Let $M_{h} \subset H^{1}(\Omega)$ be a normal finite element space with index $l$, and let $h_{c}>0$ be a spatial step of quasi-regular partition $J_{h_{c}}$. The largest diameter of tetrahedron elements or cube elements is not exceeding $h_{c}$. Approximation order is $O\left(h_{c}^{l+1}\right)$ (Ciarlet, 1978),

$$
\begin{aligned}
& \left(A_{c}\right) \quad \inf _{z_{h} \in M_{h}}\left\{\left\|z-v_{h}\right\|_{L^{2}(\Omega)}+h_{c}\left\|z-z_{h}\right\|_{H^{1}(\Omega)}\right\} \leq K h_{c}^{l+1}, \\
& \left(I_{c}\right) \quad\|w\|_{L^{\infty}(\Omega)} \leq K h_{c}^{-3 / 2}\|w\|_{L^{2}(\Omega)},\|w\|_{H^{1}(\Omega)} \leq K h_{c}^{-1}\|w\|_{L^{2}(\Omega)} .
\end{aligned}
$$

Let $\tau(X, t)$ denote a unit vector of the characteristics, and let $\psi=\left[\phi^{2}+|\mathbf{u}|^{2}\right]^{1 / 2}=\left(\phi^{2}+\sum_{i=1}^{3} u_{i}^{2}\right)^{1 / 2}$. Then the characteristic derivative is formulated by

$$
\psi \frac{\partial c}{\partial \tau}=\phi \frac{\partial c}{\partial t}+\mathbf{u} \cdot \nabla c
$$

Let $\Delta t_{c}=T / N$ denote the time step for the concentration, and $t^{n}=n \Delta t_{c} . N$ is a positive integer. For $X \in \Omega$, define

$$
\check{X}^{n-1}=X-\phi^{-1} \mathbf{u}^{n} \Delta t_{c}, \check{c}^{n-1}(X)=c^{n-1}\left(\check{X}^{n-1}\right) .
$$

The characteristic derivative, $\frac{\partial c^{n}}{\partial \tau}(X)=\frac{\partial c}{\partial \tau}\left(X, t^{n}\right)$, is approximated by a backward difference quotient

$$
\frac{\partial c^{n}}{\partial \tau}(X) \approx \frac{c^{n}(X)-\check{c}^{n-1}(X)}{\Delta t_{c} \psi^{n}}
$$

where $\psi^{n}=\left[\phi^{2}+\left|\mathbf{u}^{n}\right|^{2}\right]^{1 / 2}$.

The variation of (2) is defined as follows. A function, $c: J \rightarrow H^{1}(\Omega)$, is determined by

$$
\left(\phi \frac{\partial c}{\partial t}, z\right)+(\mathbf{u} \cdot \nabla c, z)+(\mathbf{D}(\mathbf{u}) \nabla c, \nabla z)=((\tilde{c}-c) \tilde{q}, z), \forall z \in H^{1}(\Omega), t \in J .
$$

By using (19), (22) is restated by

$$
\begin{aligned}
& \left(\psi \frac{\partial c}{\partial \tau}, z\right)+(\mathbf{D}(\mathbf{u}) \nabla c, \nabla z)=((\tilde{c}-c) \tilde{q}, z), \forall z \in H^{1}(\Omega), t \in J \\
& c(X, 0)=c_{0}(X), X \in \Omega .
\end{aligned}
$$

Since the diffusion matrix $\mathbf{D}(\mathbf{u})$ is positive semi-definite, so $L^{2}$ projection is introduced, replacing elliptic projection, to show convergence analysis. For $t \in J, \bar{c}_{h} \in M_{h}$ is defined by

$$
\left(\phi \bar{c}_{h}, \chi\right)=(\phi c, \chi), \forall \chi \in M_{h} .
$$

Then the estimate holds (Ciarlet,1978)

$$
\left\|c-\bar{c}_{h}\right\|_{L^{2}\left(L^{2}\right)}+h_{c}\left\|c-\bar{c}_{h}\right\|_{L^{2}\left(H^{1}\right)} \leq K h_{c}^{l+1}\|c\|_{L^{2}\left(H^{l+1}\right)} .
$$

The characteristic finite element scheme of (23) is constructed. $\left\{c_{h}^{n} \in M_{h}\right\}$ is computed by

$$
\begin{aligned}
& \left(\phi \frac{c_{h}^{n}-\hat{c}_{h}^{n-1}}{\Delta t_{c}}, z_{h}\right)+\left(\mathbf{D}\left(\mathbf{u}^{\mathbf{n}}\right) \nabla c_{h}^{n}, \nabla z_{h}\right)+\left(\tilde{q} c_{h}^{n}, z_{h}\right)=\left(\left(\tilde{q} \tilde{c}\left(t^{n}\right), z_{h}\right), \forall z_{h} \in M_{h},\right. \\
& c_{h}^{0}=\bar{c}_{h}^{0},
\end{aligned}
$$

where $\bar{c}_{h}^{0}$ is an $L^{2}$ projection of initial solution $c_{0}(X)$.

\subsection{The Composite Scheme}

Combining (16) and (26), we state a composite scheme to solve (1)-(4). In actual computation Darcy velocity changes more slowly than the saturation with respect to time $t$, so spatial large step is adopted for computing (16). Time interval $J$ is partitioned $0=t_{0}<t_{1}<\cdots<t_{L}=T$, with $\Delta t_{p}^{m}=t_{m}-t_{m-1}$. All the steps except for the first step $\Delta t_{p}^{1}$ are supposed to be uniform $\Delta t_{p}^{m}=\Delta t_{p}, m \geq 2$. Each pressure node $t_{m}$ is also a saturation node $t^{n}$ where $m, n$ are positive integers, and let 
$j=\Delta t_{p} / \Delta t_{c}, j_{1}=\Delta t_{p}^{1} / \Delta t_{c}$. For a function $\varphi_{m}(X)=\varphi\left(X, t_{m}\right)$ related with saturation step $t^{n}$ for $t_{m-1}<t^{n} \leq t_{m}$, we require a velocity approximation $\mathbf{u}_{h}$ in (26). If $m \geq 2$, define a linear extrapolation of $\mathbf{u}_{h, m-1}$ and $\mathbf{u}_{h, m-2}$ as follows

$$
E \mathbf{u}_{h}^{n}=\left(1+\frac{t^{n}-t_{m-1}}{t_{m-1}-t_{m-2}}\right) \mathbf{u}_{h, m-1}-\frac{t^{n}-t_{m-1}}{t_{m-1}-t_{m-2}} \mathbf{u}_{h, m-2} .
$$

If $m=1$, set $E \mathbf{u}_{h}^{n}=\mathbf{u}_{h, 0}$.

Combining (16) with (26), replacing exact solution by numerical approximations, then we can obtain full discrete coupled scheme of (1)-(4) to find $\left\{c_{h}^{n}\right\}:\left(t^{0}, t^{1}, \cdots, t^{L}\right) \rightarrow M_{h}$ and $\left\{\mathbf{u}_{h, m}, p_{h, m}\right\}:\left(t_{0}, t_{1}, \cdots, t_{L / j}\right) \rightarrow V_{h} \times W_{h}$,

$$
\begin{aligned}
& \left(\phi \frac{c_{h}^{n}-\hat{c}_{h}^{n-1}}{\Delta t_{c}}, z_{h}\right)+\left(\mathbf{D}\left(E \mathbf{u}_{h}^{n}\right) \nabla c_{h}^{n}, \nabla z_{h}\right)+\left(\tilde{q}^{n} c_{h}^{n}, z_{h}\right)=\left(\tilde{q} c_{h}^{n}, z_{h}\right), \forall z_{h} \in M_{h}, \\
& c_{h}^{0}=\bar{c}_{h}^{0}, \quad \forall X \in \Omega,
\end{aligned}
$$

$$
\begin{aligned}
& \mathcal{A}\left(c_{h, m}, \mathbf{u}_{h, m}, \mathbf{v}\right)+\mathcal{B}\left(\mathbf{v}, p_{h, m}\right)=\left(\gamma\left(c_{h, m}\right) \nabla d, \mathbf{v}\right), \forall \mathbf{v} \in V_{h}, \\
& \mathcal{B}\left(\mathbf{u}_{h, m}, w\right)=-\left(q_{m}, w\right), \forall w \in W_{h},
\end{aligned}
$$

where $\hat{c}_{h}^{n-1}(X)=c_{h}^{n-1}\left(X-\phi^{-1} E \mathbf{u}_{h}^{n} \Delta t_{c}\right)$.

The procedures of (28) and (29) run as follows.

Step 1. Given initial approximation $c_{h}^{0}$, then by (29a) and (29b) the numerical values of $\left(\mathbf{u}_{h, 0}, p_{h, 0}\right)$ are obtained.

Step 2. Applying (28a) and (28b) to find $c_{h}^{1}, c_{h}^{2}, \cdots, c_{h}^{j_{1}}$.

Step 3. By the fact of $c_{h}^{j_{1}}=c_{h, 1}$, and by (29a), (29b), we have $\left(\mathbf{u}_{h, 1}, p_{h, 1}\right)$.

Step 4. Similarly, we get the values of $c_{h}^{j_{1}+1}, c_{h}^{j_{1}+2}, \cdots, c_{h}^{j_{1}+j},\left(\mathbf{u}_{h, 2}, p_{h, 2}\right)$.

Step 5. The program runs repeatedly as above, then all the numerical solutions are obtained.

\section{Convergence Analysis}

Based on the discussions of Darcy velocity $\mathbf{u}_{h}$ and the pressure $p_{h}$, (14) and (17), convergence analysis is shown as follows. Let $\zeta=c_{h}-\bar{c}_{h}$ and $\xi=c-\bar{c}_{h}$. From (28a), (23a) $\left(t=t^{n}\right)$, (21) and (24), taking $z_{h}=\zeta^{n}$, we have

$$
\begin{aligned}
& \left(\phi \frac{\zeta^{n}-\check{\zeta}^{n-1}}{\Delta t_{c}}, \zeta^{n}\right)+\left(\mathbf{D}\left(E \mathbf{u}_{h}^{n}\right) \nabla \zeta^{n}, \nabla \zeta^{n}\right)+\left(\tilde{q}^{n} \zeta^{n}, \zeta^{n}\right) \\
& =\left(\sigma^{n}, \zeta^{n}\right)-\left(\phi \frac{\check{\xi}^{n-1}-\xi^{n-1}}{\Delta t_{c}}, \zeta^{n}\right)+\left(\tilde{q}^{n} \xi^{n}, \zeta^{n}\right)+\left(\mathbf{D}\left(E \mathbf{u}_{h}^{n}\right) \nabla \xi^{n}, \nabla \zeta^{n}\right) \\
& +\left(\phi \frac{\check{F}_{h}^{n-1}-\hat{F}_{h}^{n-1}}{\Delta t_{c}}, \zeta^{n}\right)-\left(\phi \frac{\check{\zeta}^{n-1}-\hat{\zeta}^{n-1}}{\Delta t_{c}}, \zeta^{n}\right)+\left(\left[\mathbf{D}\left(\mathbf{u}^{n}\right)-\mathbf{D}\left(E \mathbf{u}_{h}^{n}\right)\right] \nabla c^{n}, \nabla \zeta\right),
\end{aligned}
$$

where $\sigma^{n}=\left[\phi \frac{\partial c^{n}}{\partial t}+E \mathbf{u}^{n} \cdot \nabla c^{n}\right]-\phi \frac{c^{n}-\check{c}^{n-1}}{\Delta t_{c}}, F_{h}=\bar{c}_{h}, \check{\zeta}^{n-1}=\zeta^{n-1}\left(\check{X}^{n-1}\right), \cdots$.

The first term on the left-hand side of (30) is estimated. Noting that

$$
\begin{aligned}
\left(\phi \frac{\zeta^{n}-\check{\zeta}^{n-1}}{\Delta t_{c}}, \zeta^{n}\right) & \geq \frac{1}{2 \Delta t_{c}}\left\{\left(\phi \zeta^{n}, \zeta^{n}\right)-\left(\phi \check{\zeta}^{n-1}, \check{\zeta}^{n-1}\right)\right\} \\
& =\frac{1}{2 \Delta t_{c}}\left\{\left(\phi \zeta^{n}, \zeta^{n}\right)-\left(\phi \zeta^{n-1}, \zeta^{n-1}\right)\right\}+\frac{1}{2 \Delta t_{c}}\left\{\left(\phi \zeta^{n-1}, \zeta^{n-1}\right)-\left(\phi \check{\zeta}^{n-1}, \check{\zeta}^{n-1}\right)\right\}
\end{aligned}
$$

The second term of (31), $\frac{1}{2 \Delta t_{c}}\left\{\left(\phi \zeta^{n-1}, \zeta^{n-1}\right)-\left(\phi \check{\zeta}^{n-1}, \breve{\zeta}^{n-1}\right)\right\}$, is discussed. Let $Y=\check{X}=X-\mathbf{u}\left(X, t^{n}\right) \phi^{-1} \Delta t_{c} \triangleq R(X)$, and let 
$\check{\Omega}=R(\Omega)$ be the range of the mapping $R$. Since $\operatorname{det} D R(X)=1-\nabla \cdot\left(\frac{\mathbf{u}}{\phi}\right) \Delta t_{c}+O\left(\left(\Delta t_{c}\right)^{2}\right)$, then

$$
\begin{aligned}
& \frac{1}{2 \Delta t_{c}}\{(\phi \breve{\zeta}, \breve{\zeta})-(\phi \zeta, \zeta)\} \\
= & \frac{1}{2 \Delta t_{c}}\left\{\int_{\check{\Omega}} \phi(x) \zeta(y) \zeta(y)(\operatorname{det} D R(X))^{-1} d y-\int_{\Omega} \phi(X) \zeta(x) \zeta(x) d x\right\} \\
= & \frac{1}{2 \Delta t_{c}}\left\{\int_{\check{\Omega}} \phi(x) \zeta(y) \zeta(y)\left[1+\nabla \cdot\left(\frac{\mathbf{u}}{\phi}\right) \Delta t_{c}+O\left(\left(\Delta t_{c}\right)^{2}\right)\right] d y-\int_{\Omega} \phi(x) \zeta(x) \zeta(x) d x\right\} \\
= & \frac{1}{2 \Delta t_{c}}\left\{\int_{\check{\Omega}} \phi(y) \zeta(y) \zeta(y) d y-\int_{\Omega} \phi(X) \zeta(x) \zeta(x) d x+\int_{\check{\Omega}}(\phi(x)-\phi(y)) \zeta(y) \zeta(y) d y\right. \\
& \left.+\int_{\check{\Omega}} \phi(x) \zeta(y) \zeta(y)\left[\nabla \cdot\left(\frac{\mathbf{u}}{\phi}\right) \Delta t_{c}+O\left(\left(\Delta t_{c}\right)^{2}\right)\right] d y\right\} \\
= & \frac{1}{2 \Delta t_{c}}\left\{\int_{\check{\Omega} \mid \Omega} \phi(y) \zeta(y) \zeta(y) d y-\int_{\Omega \mid \check{\Omega}} \phi(x) \zeta(x) \zeta(x) d x\right\} \\
& +\frac{1}{2 \Delta t_{c}}\left\{\int_{\check{\Omega}}(\phi(x)-\phi(y)) \zeta(y) \zeta(y) d y+\int_{\check{\Omega}} \phi(x) \zeta(y) \zeta(y)\left[\nabla \cdot\left(\frac{\mathbf{u}}{\phi}\right) \Delta t_{c}+O\left(\left(\Delta t_{c}\right)^{2}\right)\right] d y\right\} \\
= & T_{1}+T_{2} .
\end{aligned}
$$

From the boundary condition $\mathbf{u} \cdot v=0$, we can get meas $\{\check{\Omega} \backslash \Omega\}=O\left(\left(\Delta t_{c}\right)^{2}\right)$, meas $\{\Omega \backslash \check{\Omega}\}=O\left(\left(\Delta t_{c}\right)^{2}\right)$. If the partition satisfies $\Delta t_{c}=O\left(h_{c}^{3}\right)$, then $T_{1}$ is bounded by $\left|T_{1}\right| \leq K\left\|\phi^{1 / 2} \zeta\right\|_{L^{\infty}}^{2} \Delta t_{c} \leq K h_{c}^{-3} \Delta t_{c}(\phi \zeta, \zeta) \leq K(\phi \zeta, \zeta)$. Similarly, $T_{2}$ is bounded by $\left|T_{2}\right| \leq K(\phi \zeta, \zeta)$. Then,

$$
\frac{1}{2 \Delta t_{c}}\{(\phi \check{\zeta}, \check{\zeta})-(\phi \zeta, \zeta)\} \leq K(\phi \zeta, \zeta) .
$$

Estimate the other terms on the left-hand side of (30),

$$
\begin{gathered}
\left(\mathbf{D}\left(E \mathbf{u}_{h}^{n}\right) \nabla \zeta^{n}, \nabla \zeta^{n}\right)=\left\|\mathbf{D}^{1 / 2}\left(E \mathbf{u}_{h}^{n}\right) \nabla \zeta^{n}\right\|_{0}^{2} . \\
\left(\tilde{q} \zeta^{n}, \zeta^{n}\right)=\left\|\tilde{q}^{1 / 2} \zeta^{n}\right\|_{0}^{2} .
\end{gathered}
$$

The right-hand side of (30) is considered as follows,

$$
\begin{aligned}
& \left|\left(\sigma^{n}, \zeta^{n}\right)\right| \leq K\left\{\Delta t_{c}\left\|\frac{\partial^{2} c}{\partial \tau^{2}}\right\|_{L^{2}\left(t^{n-1}, t^{n} L^{2}\right)}^{2}+\left\|\zeta^{n}\right\|^{2}\right\} . \\
& \left|\left(\phi \frac{\check{\xi}^{n-1}-\xi^{n-1}}{\Delta t_{c}}, \zeta^{n}\right)\right| \leq K\left\{\left\|\nabla \xi^{n-1}\right\|^{2}+\left\|\zeta^{n}\right\|^{2}\right\} \leq K\left\{h^{2 l}+\left\|\zeta^{n}\right\|^{2}\right\} . \\
& \left|\left(\mathbf{D}\left(E \mathbf{u}_{h}^{n}\right) \nabla \xi^{n}, \nabla \zeta^{n}\right)\right| \leq \frac{1}{3}\left\|\mathbf{D}^{1 / 2}\left(E \mathbf{u}_{h}^{n}\right) \nabla \zeta^{n}\right\|_{0}^{2}+K h^{2 l} . \\
& \left|\left(\phi \frac{\check{F}_{h}^{n-1}-\hat{F}_{h}^{n-1}}{\Delta t_{c}}, \zeta^{n}\right)\right| \leq K\left\|\nabla \bar{c}_{h}^{n-1}\right\|_{\infty}\left\|E\left(\mathbf{u}-\mathbf{u}_{h}^{n}\right)\right\|_{0}\left\|\zeta^{n}\right\|_{0} \\
& \leq K\left\{h_{c}^{2(l+1)}+h_{p}^{2(k+1)}+\left(\Delta t_{c}\right)^{2}+\left\|\zeta_{m}\right\|^{2}+\left\|\zeta_{m-1}\right\|^{2}+\left\|\zeta^{n}\right\|^{2}\right\} . \\
& \left|\left(\phi \frac{\check{\zeta}^{n-1}-\hat{\zeta}^{n-1}}{\Delta t_{c}}, \zeta^{n}\right)\right| \leq K\left\|\nabla \zeta ^ { n - 1 } \left|\left\|\mid E\left(\mathbf{u}-\mathbf{u}_{h}^{n}\right)\right\|_{0}\left\|\zeta^{n}\right\|_{\infty}\right.\right. \\
& \leq K h_{c}^{-5 / 2}\left\|E\left(\mathbf{u}-\mathbf{u}_{h}^{n}\right)\right\|_{0}\left\|\zeta^{n-1}\right\|_{0}\left\|\zeta^{n}\right\|_{0} \\
& \leq K\left\{h_{c}^{2(l+1)}+h_{p}^{2(k+1)}+\left\|\zeta_{m}\right\|^{2}+\left\|\zeta_{m-1}\right\|^{2}+\left\|\zeta^{n-1}\right\|^{2}\right\} .
\end{aligned}
$$

An induction hypothesis is used

$$
\sup _{0 \leq n \leq L-1} h_{c}^{-5 / 2}\left\|\zeta^{n}\right\|_{0} \leq K
$$

The last term is discussed.

$$
\begin{aligned}
& \left|\left(\left[\mathbf{D}\left(\mathbf{u}^{n}\right)-\mathbf{D}\left(E \mathbf{u}_{h}^{n}\right)\right] \nabla c^{n}, \nabla \zeta\right)\right| \\
& =\int_{\Omega} \int_{0}^{1}\left[\frac{\partial \mathbf{D}}{\partial \mathbf{u}}\left(\theta \mathbf{u}^{n}-(1-\theta) E \mathbf{u}_{h}^{n}\right)-\frac{\partial \mathbf{D}}{\partial \mathbf{u}}\left(E \mathbf{u}_{h}^{n}\right)\right] d \theta\left(\mathbf{u}^{n}-E \mathbf{u}_{h}^{n}\right) \nabla c^{n} \cdot \nabla \zeta^{n} d X \\
& \quad+\int_{\Omega} \frac{\partial \mathbf{D}}{\partial \mathbf{u}}\left(E \mathbf{u}_{h}^{n}\right)\left(\mathbf{u}^{n}-E \mathbf{u}_{h}^{n}\right) \nabla c^{n} \cdot \nabla \zeta^{n} d X=W_{1}+W_{2} .
\end{aligned}
$$


Suppose that $\frac{\partial^{2} \mathbf{D}}{\partial \mathbf{u}^{2}}$ is bounded, we have

$$
\begin{aligned}
\left|W_{1}\right| & \leq K \mid \frac{\partial^{2} \mathbf{D}}{\partial \mathbf{u}^{2}}\left\|\mathbf{u}^{n}-E \mathbf{u}_{h}^{n}\right\|_{0}^{2}\|\nabla \zeta\|_{\infty} \\
& \leq K h_{c}^{-5 / 2}\left\{\left(\Delta t_{c}\right)^{2}+h_{c}^{2(l+1)}+h_{p}^{2(k+1)}+\left\|\zeta_{m}\right\|^{2}+\left\|\zeta_{m-1}\right\|^{2}\right\}\left\|\zeta^{n}\right\|_{0} \\
& \leq K\left\{\left(\Delta t_{c}\right)^{2}+h_{c}^{2(l+1)}+h_{p}^{2(k+1)}+\left\|\zeta_{m}\right\|^{2}+\left\|\zeta_{m-1}\right\|^{2}\right\},
\end{aligned}
$$

and

$$
\left|W_{2}\right| \leq \frac{1}{3}\left\|\mathbf{D}^{1 / 2}\left(E \mathbf{u}_{h}^{n}\right) \nabla \zeta^{n}\right\|_{0}^{2}+K \int_{\Omega} \mathbf{D}\left(E \mathbf{u}_{h}^{n}\right)^{-1}\left|\frac{\partial \mathbf{D}}{\partial \mathbf{u}}\left(E \mathbf{u}_{h}^{n}\right)\right|^{2}\left|\mathbf{u}^{n}-E \mathbf{u}_{h}^{n}\right|^{2}\left|\nabla c^{n}\right|^{2} d X .
$$

Later it is shown that $\mathbf{D}^{-1}\left|\frac{\partial \mathbf{D}}{\partial \mathbf{u}}\right|^{2}$ is bounded. For simplicity, we suppose that $\mathbf{u}$ is oriented in $x$-direction, since the rotation transformation of coordinates will not affect the size of $\left|\frac{\partial \mathbf{D}}{\partial \mathbf{u}}\right|^{2}$. Then,

$$
\begin{aligned}
& \mathbf{D}=\mathbf{D}_{m}+|\mathbf{u}|^{\beta}\left(\begin{array}{lll}
\alpha_{l} & 0 & 0 \\
0 & \alpha_{t} & 0 \\
0 & 0 & \alpha_{t}
\end{array}\right), \quad \mathbf{D}^{-1} \leq|\mathbf{u}|^{-\beta}\left(\begin{array}{lll}
\alpha_{l}^{-1} & 0 & 0 \\
0 & \alpha_{t}^{-1} & 0 \\
0 & 0 & \alpha_{t}^{-1}
\end{array}\right), \\
& \frac{\partial \mathbf{D}}{\partial u_{x}}=|\mathbf{u}|^{\beta-1}\left(\begin{array}{lll}
\alpha_{l} & 0 & 0 \\
0 & \alpha_{t} & 0 \\
0 & 0 & \alpha_{t}
\end{array}\right), \quad \frac{\partial \mathbf{D}}{\partial u_{y}}=|\mathbf{u}|^{\beta-1}\left(\begin{array}{lll}
0 & \alpha_{l}-\alpha_{t} & 0 \\
\alpha_{l}-\alpha_{t} & 0 & 0 \\
0 & 0 & 0
\end{array}\right), \\
& \frac{\partial \mathbf{D}}{\partial u_{z}}=|\mathbf{u}|^{\beta-1}\left(\begin{array}{lll}
0 & 0 & \alpha_{l}-\alpha_{t} \\
0 & 0 & 0 \\
\alpha_{l}-\alpha_{t} & 0 & 0
\end{array}\right), \\
& \left|\mathbf{D}^{-1}\left(\frac{\partial \mathbf{D}}{\partial \mathbf{u}}\right)^{2}\right| \leq|\mathbf{u}|^{\beta-2}\left\{\beta^{2}\left(\begin{array}{lll}
\alpha_{l} & 0 & 0 \\
0 & \alpha_{t} & 0 \\
0 & 0 & \alpha_{t}
\end{array}\right)+\left(\begin{array}{lll}
\alpha_{l}^{-1}\left(\alpha_{l}-\alpha_{t}\right)^{2} & 0 & 0 \\
0 & \alpha_{t}^{-1}\left(\alpha_{l}-\alpha_{t}\right)^{2} & 0 \\
0 & 0 & 0
\end{array}\right)\right. \\
& \left.+\left(\begin{array}{lll}
\alpha_{l}^{-1}\left(\alpha_{l}-\alpha_{t}\right)^{2} & 0 & 0 \\
0 & 0 & 0 \\
0 & 0 & \alpha_{t}^{-1}\left(\alpha_{l}-\alpha_{t}\right)^{2}
\end{array}\right)\right\}
\end{aligned}
$$

When the constraint conditions hold,

$$
\frac{\alpha_{l}^{2}}{\alpha_{t}} \leq \alpha^{*}<\infty, \quad \frac{\alpha_{t}^{2}}{\alpha_{l}} \leq \alpha^{*}<\infty, \quad \beta \geq 2,
$$

then $\mathbf{D}^{-1}\left|\frac{\partial \mathbf{D}}{\partial \mathbf{u}}\right|^{2}$ is bounded. Now $\frac{\partial^{2} \mathbf{D}}{\partial \mathbf{u}^{2}}$ is argued. Noting that

$$
\begin{aligned}
\frac{\partial^{2} \mathbf{D}}{\partial u_{x}^{2}}=\beta(\beta-1)|\mathbf{u}|^{\beta-2}\left(\begin{array}{lll}
\alpha_{l} & 0 & 0 \\
0 & \alpha_{t} & 0 \\
0 & 0 & \alpha_{t}
\end{array}\right), & \frac{\partial^{2} \mathbf{D}}{\partial u_{y}^{2}}=2 \mid \mathbf{u}^{\beta-2}\left(\begin{array}{lll}
\alpha_{t} & 0 & 0 \\
0 & \alpha_{l} 0 & 0 \\
0 & 0 & \alpha_{t}
\end{array}\right), \\
\frac{\partial^{2} \mathbf{D}}{\partial u_{z}^{2}}=2 \mid \mathbf{u}^{\beta-2}\left(\begin{array}{lll}
\alpha_{l} & 0 & 0 \\
0 & \alpha_{t} & 0 \\
0 & 0 & \alpha_{t}
\end{array}\right), & \frac{\partial^{2} \mathbf{D}}{\partial u_{x} \partial u_{y}}=\beta \mid \mathbf{u}^{\beta-2}\left(\begin{array}{lll}
0 & \alpha_{l}-\alpha_{t} & 0 \\
\alpha_{l}-\alpha_{t} & 0 & 0 \\
0 & 0 & 0
\end{array}\right), \cdots
\end{aligned}
$$

we can get that $\frac{\partial^{2} \mathbf{D}}{\partial \mathbf{u}^{2}}$ is bounded for $\beta \geq 2$. In a similar discussion, $W_{2}$ is estimated by the right-hand side expression of (43a).

For (30), using the estimates of (31)-(43), we have

$$
\begin{aligned}
& \frac{1}{2 \Delta t_{c}}\left\{\left\|\phi^{1 / 2} \zeta^{n}\right\|_{0}^{2}-\left\|\phi^{1 / 2} \zeta^{n-1}\right\|_{0}^{2}\right\}+\left\|\mathbf{D}^{1 / 2}\left(E \mathbf{u}_{h}^{n}\right) \nabla \zeta^{n}\right\|_{0}^{2}+\left\|\tilde{q}^{1 / 2} \zeta^{n}\right\|_{0}^{2} \\
& \leq K\left\{\Delta t_{c}\left\|\frac{\partial^{2} c}{\partial \tau^{2}}\right\|_{L^{2}\left(t^{n-1}, t^{n} ; L^{2}\right)}^{2}+\left(\Delta t_{c}\right)^{2}+\left(\Delta t_{p}^{1}\right)^{3}+\left(\Delta t_{p}\right)^{4}+h_{c}^{2 l}+h_{p}^{2(k+1)}\right. \\
& \left.\quad+\left\|\zeta_{m-1}\right\|^{2}+\left\|\zeta_{m}\right\|^{2}+\left\|\zeta^{n-1}\right\|^{2}+\left\|\zeta^{n}\right\|^{2}\right\} .
\end{aligned}
$$

Multiplying both sides of (44) by $2 \Delta t$, summing them over $n(1 \leq n \leq L)$, and applying the discrete Gronwall inequality, we have

$$
\left\|\zeta^{L}\right\|_{0}^{2} \leq K\left\{h_{c}^{2 l}+h_{p}^{2(k+1)}+\left(\Delta t_{c}\right)^{2}+\left(\Delta t_{p}^{1}\right)^{3}+\left(\Delta t_{p}\right)^{4}\right\}
$$


It remains to testify the induction hypothesis (41). As $l \geq 3$, and the partition parameters satisfy

$$
\Delta t_{c}=O\left(h_{c}^{5 / 2}\right), \quad h_{p}^{k+1}=O\left(h_{c}^{5 / 2}\right),
$$

then it is easy to check the proof of (41).

The following statement is concluded by using (17), (14), (45) and (25).

Theorem. Suppose that the problem of (1)-(4) is regular (R), and positive semi-definite (C). And suppose that the index of $M_{h}, l \geq 3$, and (46) holds. Adopting the schemes of (28) and (29), we have the following estimates,

$$
\left\|\mathbf{u}-\mathbf{u}_{h}\right\|_{L^{\infty}(H(\mathrm{div}))}+\left\|p-p_{h}\right\|_{L^{\infty}\left(L^{2}\right)}+\left\|c-c_{h}\right\|_{L^{\infty}\left(L^{2}\right)} \leq K\left\{h_{p}^{k+1}+h_{c}^{l}+\Delta t_{c}+\left(\Delta t_{p}^{1}\right)^{3 / 2}+\left(\Delta t_{p}\right)^{2}\right\},
$$

where $K$ is a constant dependent on $p, c$ and their derivatives, independent of the parameters $\Delta t_{c}, h_{p}, h_{c}$.

\section{Conclusions and Discussions}

In the present paper, we discuss a mixed element-characteristic finite element method to solve three-dimensional incompressible miscible positive semi-definite displacement problem in porous media. In $\S 1$ Introduction, we state the mathematical model, physical background and some related international research studies. In $\S 2$, we define some notations and partitions, then construct a composite computational scheme of mixed element-characteristic finite element. In $\S 3$, we introduce an induction hypothesis and use theoretical techniques of priori estimates, then show convergence analysis of the present scheme. In conclusion, the numerical scheme and its theoretical analysis can solve actual applications such as well-known positive semi-definite displacement problem efficiently.

\section{Acknowledgements}

The authors express their deep appreciation to Prof. J. Douglas Jr, Prof. R. E. Ewing, and Prof. Jiang Lishang for their many helpful suggestions in the serial research of numerical simulation of energy sciences.

\section{References}

Brezzi, F. (1974). On the existence, uniqueness and approximation of saddle-point problems arising from lagrangian multipliers. RAIRO Anal. Numer., 2, 129-151. https://doi.org/10.1051/m2an/197408r201291

Ciarlet, P. G. (1978). The finite element method for elliptic problems. Amsterdam: North-Holland.

Dawson, C. N., Russell, T. F., \& Wheeler, M. F. (1989). Some improved error estimates for the modified method of characteristics. SIAM J. Numer. Anal., 26(6), 1487-1512. https://doi.org/10.1137/0726087

Douglas, Jr. J. (1983). Finite difference method for two-phase in compressible flow in porous media. SIAM J. Numer. Anal., 20(4), 681-696. https://doi.org/10.1137/0720046

Douglas, Jr. J., Ewing, R. E., \& Wheeler, M. F. (1983). Approximation of the pressure by a mixed method in the simulation of miscible displacement. RAIRO Anal. Numer., 17(1), 17-33. https://doi.org/10.1051/m2an/1983170100171

Douglas, Jr. J., Ewing, R. E., \& Wheeler, M. F. (1983). A time-discretization procedure for a mixed finite element approximation of miscible displacement in porous media. RAIRO Anal. Numer., 17(3), 249-265. https://doi.org/10.1051/m2an/1983170302491

Ewing, R. E. (1983). The Mathematics of Reservoir Simulation. SIAM, Philadelphia. https://doi.org/10.1137/1.9781611971071

Ewing, R. E., Russell, T. F., \& Wheeler, M. F. (1984). Convergence analysis of an approximation of miscible displacement in porous media by mixed finite elements and a modified method of characteristics. Comp. Meth. Appl. Mech. Eng., 47(1-2), 73-92. https://doi.org/10.1016/0045-7825(84)90048-3

Raviart, P. A., \& Thomas, J. M. (1977). A mixed finite element method for second order elliptic problems, in: Mathematical Aspects of the Finite Element Method. Lecture Notes in Mathematics, 606, 292-315, Berlin: Springer-Verlag. https://doi.org/10.1007/BFb0064470

Russell, T. F. (1985). Time stepping along characteristics with incomplete interaction for a Galerkin approximation of miscible displacement in porous media. SLAM J. Numer. Anal., 22(5), 970-1013. https://doi.org/10.1137/0722059

Thomase, J. M. (1977). Sur L'analyse numerique des methods d'elements finis hybrides et mixtes. These. Universte Pierre et Marie Curie.

Yuan, Y. R. (1997). Characteristic finite element scheme and analysis the three-dimensional two-phase displacement semi-definite problem. Chin. Sci. Bull., 1, 17-32. https://doi.org/10.1007/BF02882512 
Yuan, Y. R. (1999). Characteristic finite difference methods for positive semidefinite problem of two phase miscible flow in porous media. J. Systems Sci. Math. Sci., 12(4), 299-306.

Yuan, Y. R., \& Han, Y. J. (2008). Numerical simulation of migration-accumulation of oil resources. Comput. Geosci., 12, 153-162. https://doi.org/10.1007/s10596-007-9075-5

Yuan, Y. R., Wang, W. Q., \& Han, Y. J. (2010). Theory, method and application of a numerical simulation oil resources basin methods of numerical solution of aerodynamic problems. Special Topic E Reviews in Porous Media-An international Journal, 1(1), 49-66.

Yuan, Y. R. (2013). Theory and application of reservoir numerical simulation. Science Press, Beijing.

Yuan, Y, R., Cheng, A. J., Yang, D. P., \& Li, C. F. (2014). Theory and application of numerical simulation of chemical flooding in high temperature and high salt reservoirs. International Journal of Geosciences, 5, 956-970.

Yuan, Y, R., Cheng, A. J., Yang, D. P., \& Li, C. F. (2015). Applications, theoretical analysis, numerical method and mechanical model of the polymer flooding in porous media. Special Topics $\mathcal{E}$ Reviews in Porous Media-An International Journal. 6(4), 383-401. https://doi.org/10.1615/SpecialTopicsRevPorousMedia.v6.i4.60

\section{Copyrights}

Copyright for this article is retained by the author(s), with first publication rights granted to the journal.

This is an open-access article distributed under the terms and conditions of the Creative Commons Attribution license (http://creativecommons.org/licenses/by/4.0/). 